\title{
The Role of Gut Microbiota in Gastrointestinal Disease
}

\section{Riddhima Banerji*}

MSc in Gastroenterology, Bart's and The London School of Medicine, Queen Mary University of London

*Corresponding Author: Riddhima Banerji, MSc in Gastroenterology, Bart's and The London School of Medicine, Queen Mary University of London.
Received: March 29, 2021

Published: May 07, 2021

(C) All rights are reserved by Riddhima

Banerji.

\begin{abstract}
Multiple studies have demonstrated that microorganisms in the gut play a key role in maintaining the normal function of the gastrointestinal tract. They are a diverse group of organisms which share an intricate relationship with the gut and one another, thereby helping to maintain a delicate physiological balance. Changes in the internal, cellular or external environment can disrupt this balance and result in pathological changes and disease. The last decade has witnessed important developments in the understanding of the role of gut microbiota in the genesis of gastrointestinal disease. The recent pandemic has underscored their significant function. Therefore, it is vital that research in this crucial area continues.
\end{abstract}

Keywords: Gut Microbiota; Gastrointestinal Disease; Gastrointestinal Tract

\section{Introduction}

The gastrointestinal tract contains a diverse group of microorganisms which are now believed to have a key role in maintaining health. Studies have demonstrated that the gut is home to 1000 bacterial species and contains genes which are a 100 times greater in number than those in the human genome. These bacteria share a dynamic relationship with gut, which is constantly evolving and changing in response to internal (genetics, stress, aryl hydrocarbon receptor and increasing age) and external triggers (diet, pollution, antibiotic use, breast-feeding). They play a vital role in metabolism, nutrition, physiology and immune function. In the intestine, they restrict the growth of pathogens by modulating the development of intestinal immune cells, such as Th17 cells, regulatory $\mathrm{T}$ cells and B cells. With regard to metabolic function, the gut bacteria produces short chain fatty acids (SCFAs), such as butyrate, from dietary fibre, which augments the epithelial cell barrier and promotes the multiplication of anti-inflammatory cells. Interestingly, with regard to bile acid metabolism in the intestine, metabolism by gut microbiota can have both positive and negative effects. For ex- ample, the production of secondary bile acids such as deoxycholic acid (DCA) brings about destruction of the intestinal mucosa and DNA damage. However, paradoxically, there are some secondary bile acids that decrease the growth of virulent organisms.

Various studies indicate that disruption of the gut microbiota may be related to the development of gastrointestinal diseases such as inflammatory bowel disease, irritable bowel syndrome, coeliac disease, colorectal cancer, infectious colitis and functional dyspepsia $[17,22]$. This essay will discuss the pathogenic role of gut microbiota in gastrointestinal disease.

\section{Objective of the Study}

To evaluate the role of gastrointestinal microbiota in the pathogenesis of disease.

\section{Methods}

Multiple studies were critically analysed. An in depth evaluation of each study was carried out in order to determine the function of gut microbiota. 


\section{Inflammatory bowel disease}

Studies indicate that the diversity of gut microbiota is significantly reduced in IBD $[5,14]$. There is evidence to show that a disruption in NLRP (NOD like receptor family pyrin domain containing protein) signalling predisposes to disturbances in the functioning of intestinal microbiota. Nlrp6 deficient mice have a disproportionately higher number of the phyla Prevotellaceae, which damages the barrier function of the intestinal mucosa [13,32]. Nlrp6 deficiency stimulates CCL5, leading to inflammation of the intestine due to infiltration if immune cells. These bacteria also have a significant presence in the intestines of patients with IBD.

Similarly, deficiency of the NOD 2 gene (which has a known association with Crohn's disease) in mice is associated with a disruption in the gut microbiota and a heightened predisposition to dextran sulphate sodium (DSS) induced colitis [3].

Similar experiments with mice deficient in IL-10 or IL-2 genes have been used as models for colitis similar to IBD [26,28], showing that enteric bacteria are required for the development of spontaneous colitis in these mouse populations. These findings indicate that gut microbiota is a potent instigator of intestinal inflammation. Thus, there is an association between the genetic background of an individual and the functioning of gut microbiota.

There are several environmental triggers for IBD. Significant among these is the role of diet in inducing dysbiosis. For example, it has been shown that taurocholic acid from milk-derived fats stimulated the growth of a commensal bacterium, Bilophila wadsworthia, which produces hydrogen sulphate, a substance that triggers inflammation [4]. Similarly, the number of bacteria such as Erysipelotrichaceae as well as Bacteroides fragilis were increased in diets high in fat and beef [8,33]. These bacteria are thought to be potential pathogens, with a possible role in the development of IBD. A high level of protein ingestion produces substances in the colon that promote the overgrowth of bacteria such as Desulfovibrio and Desulfuromonas, which are observed to have a more significant presence in the colon of IBD patients, as compared to healthy individuals [24].

Smoking is also believed to have an effect on gut bacteria. A study has shown that Anaerostipes, a type of bacteria that changes lactate to butyrate, was decreased in subjects with IBD who smoked as opposed to those who did not [16]. This chemical change is signifi- cant as butyrate is responsible for strengthening intestinal epithelial barrier function and expediting regulatory $\mathrm{T}$ cell development. Thus, smoking may result in reduced butyrate production by gut bacteria and enhance the propensity of the host to developing IBD.

Antibiotics have been demonstrated to have a major impact on the composition on the gut microbiota, which induces bacterial overgrowth. Among antibiotics, ciprofloxacin and metronidazole are notorious for disturbing the composition of commensal bacteria, especially reducing the number of Dorea, Butyricicoccus and Coriobacteriaceae in paediatric IBD patients [12]. The proliferation of pathogens such as Clostridium difficile is also triggered by the use of antibiotics. This is a notable point, as $C$. difficile induced colitis is responsible for increased morbidity in hospitals on addition to being an exacerbating factor as well as a grave complication of patients with IBD.

\section{Colorectal cancer}

Alteration in the in the composition and distribution of microbiota in the colon may be instrumental in the development and advancement of colon carcinoma. The Bacteroides fragilis toxin, in particular, produced by enterotoxigenic B. fragilis (ETBF), enhances the permeability of the colonic epithelial barrier and accelerates the Wnt and Beta-catenin signalling pathways, thereby leading to a proliferation of malignant cells in the colon. This is brought about by the cleavage of the E-cadherin a tumour suppressor protein as well as a change in the morphology of colon epithelial cells, both of which are effects of the toxin [23].

Studies have demonstrated that excessive quantities of an invasive strain of the Fusobacterium nucleatum (an ordinarily commensal bacteria in the oral cavity but having a pathogenic effect in the gut) triggers the formation of colonic tumours through the regulation of E-cadherin and Beta catenin pathways, using an adhesion protein called FadA [25].

\section{Coeliac disease}

Research has shown that a disruption in the normal constitution of the gut microbiota may have a role in the genesis of coeliac disease. Studies have reported that bacteria such as Klebsiella oxytoca, Staphylococcus epidermidis and Staphylococcus pasteuri are found in abundance in the duodenum of individuals with ongoing coeliac disease as compared to healthy individuals. In comparison, bacteria such as Streptococcus anginosus and Streptococcus mutans 
are found to be in lesser quantity in those with the disease as compared to healthy subjects, irrespective of the inflammation status [27]. Genetic mutations, such as that of the FUT 2 gene (fucosyltransferase 2) have been associated with the pathogenesis of coeliac disease. In the context of the role gut microbiota in the development of GI disease, this is relevant in that FUT 2 mutations have an adverse impact on the bacterial diversity in the gut. Specifically, this genetic mutation reduces the quantity of the Bifidobacterium species in the gut, a development which may lead to proliferation of the fungus Candida albicans and a subsequent imbalance of the gut flora.

The pathogenic role of intestinal microbiota in coeliac disease was further underscored by a study by Galipeau and McCarville which demonstrated that gluten-sensitive NOD/DQ8 mice who underwent antibiotic treatment (with vancomycin), triggered gluten-induced pathology in the immune system. Interestingly, a subsequent reduction in faecal microbial diversity was also observed. The study observed that a disturbance in the balance of the microbiota had taken place, with an increase in the abundance of Proteobacteria, such as Escherichia and Helicobacter species and a notable reduction of the Bacteroides and Parabacteroides species.

Recent evidence has emerged suggesting that reduced quantities of the Bifidobacterium species may be associated with a higher risk of developing autoimmune diseases. This was indicated by a study that demonstrated that these bacteria are in reduced quantity in the breast milk of mothers with coeliac disease, thereby implying that infants born to mothers with coeliac disease may also have a decreased abundance of Bifidobacterium [21]. Thus, these infants may be predisposed to developing coeliac disease in the future and breastfeeding may, therefore, have a protective role. The microbiota, therefore, have a prominent, protective role in coeliac disease and a disturbance of their natural diversity and composition may well have adverse effects on gut immunity.

\section{Irritable bowel syndrome}

There is growing evidence to prove that gut microbiota play a role in the pathogenesis of IBS, particularly in the area of intestinal permeability, gut motility malfunction and visceral pain responses [20]. Whether intestinal dysbiosis is a causative factor for IBS or simply a result of the disease, remains to be confirmed. However, a study has shown that IBS patients have higher numbers of Ruminococcaceae and Clostridium cluster XIVa and reduced quantities of Bacteroides as compared to healthy subjects.
Imbalance in gut microbiota may also compromise luminal metabolic function in IBS patients. Methane producing bacteria such as Methanobrevibacter smithii have been shown to be in greater quantities in the faeces of patients with constipation-dominant IBS [11]. This is a notable point, as methane has been shown to have an adverse impact on gastrointestinal motility.

Intestinal infections with disease producing bacteria such as Campylobacter, Salmonella and Shigella have been implicated in the development of IBS. There are multiple mechanisms through which these bacteria may cause IBS, two of which are a disturbance in GI motility and dysbiosis triggered by the infection itself [1].

\section{Role of gastric microbiota in functional dyspepsia}

Two Japanese studies have demonstrated that there exists an alteration in the microbiota of the gastric fluid of patients with functional dyspepsia (FD). This was specified by the predominance of Bacteroidetes relative to Proteobacteria and the absence of Acidobacteria in the gastric fluid of FD patients. These changes in bacterial composition were thought to be brought about by the reflux of small intestinal contents (along with bile acids and intestinal bacteria) into the stomach and are believed to be part of the reason for the pathophysiology in FD [9]. Another study showed an inverse relationship between the abundance of the bacteria Prevotella and the gravity of post-prandial distress-like symptoms in FD patients who were given yogurt containing a probiotic strain of Lactobacillus gasseri. The study concluded that the dominance of the bacteria Prevotella in gastric fluid could be used as a biomarker to assess the efficacy of treatment in FD [19]. Such studies underscore the role of microbiota in the pathogenesis of functional dyspepsia.

Therapeutic implications of the role of gut microbiota in GI disease

A shift in the phenotype of commensal gut bacteria can result in the development of a spectrum of GI diseases by having an adverse impact on the gut immune response [2]. A practical application of this concept in therapeutics is the introduction of healthy faecal microbiota transplant in the treatment of IBD. A study showed that IBD patients experienced a relief of $C$. difficile associated symptoms following treatment with FMT [31]. Similarly, administration of the bacteria Lactobacillus johnsonii was shown to regulate immune pathways and reduce colonization by pathobionts in the gut of CRC patients [7]. A study [10] demonstrated that the bacteria Lactobacillus casei prevented the re-growth of colorectal tumours in CRC patients, for a minimum of 4 years after treatment. 


\section{Conclusion}

It can be concluded, therefore, that gut microbiota have a significant role in the pathogenesis of gastrointestinal disease.

\section{Bibliography}

1. Beatty JK., et al. "Post-infectious irritable bowel syndrome: mechanistic insights into chronic disturbances following enteric infection". World Journal of Gastroenterology 20 (2014): 3976-3985.

2. Chow J., et al. "Pathobionts of the gastrointestinal microbiota and inflammatory disease". Current Opinion in Immunology 23 (2011): 473-480.

3. Couturier-Maillard A., et al. "NOD2-mediated dysbiosis predisposes mice to transmissible colitis and colorectal cancer". Journal of Clinical Investigation 123 (2013): 700-711.

4. Devkota S., et al. "Dietary-fat-induced taurocholic acid promotes pathobiont expansion and colitis in Il10-/- mice". Nature 487 (2012): 104-108.

5. Frank DN., et al. "Molecular-phylogenetic characterization of microbial community imbalances in human inflammatory bowel diseases". Proceedings of the National Academy of Sciences of the United States of America 104 (2007): 13780-13785.

6. Galipeau HJ., et al. "Intestinal microbiota modulates gluteninduced immunopathology in humanized mice". The American Journal of Pathology 185 (2015): 2969-2982.

7. Gianotti L., et al. "A randomized double blind trial on perioperative administration of probiotics in colorectal cancer patients". World Journal of Gastroenterology 16 (2010): 167-175.

8. Hentges DJ., et al. "Effect of a high-beef diet on the fecal bacterial flora of humans". Cancer Research 37 (1977): 568-571.

9. Igarashi M., et al. "Alteration in the gastric microbiota and its restoration by probiotics in patients with functional dyspepsia”. BMJ Open Gastroenterology (2017).

10. Ishikawa H., et al. "Randomized trial of dietary fiber and Lactobacillus casei administration for prevention of colorectal tumors". International Journal of Cancer 116 (2005): 762-767.

11. Kim G., et al. "Methanobrevibacter smithii is the predominant methanogen in patients with constipation-predominant IBS and methane on breath". Digestive Diseases and Sciences 57 (2012): 3213-3218.
12. Kronman MP., et al. "Antibiotic exposure and IBD development among children: a population-based cohort study". Pediatrics 130 (2012): e794-e803.

13. Lucke K., et al. "Prevalence of Bacteroides and Prevotella spp. in ulcerative colitis". Journal of Medical Microbiology 55 (2006): 617-624.

14. Manichanh C., et al. "The gut microbiota in IBD”. Nature Reviews Gastroenterology and Hepatology 9 (2012): 599-608.

15. Mårild K., et al. "Antibiotic exposure and the development of coeliac disease: a nationwide case-control study". BMC Gastroenterology 13 (2013): 109.

16. Morgan., et al. "Dysfunction of the intestinal microbiome in inflammatory bowel disease and treatment". Genome Biology 13 (2012): R79.

17. Mukherjee PK., et al. "Microbiota in gastrointestinal diseases". Nature Reviews Gastroenterology and Hepatology 12 (2015): 77-87.

18. Nagao-Kitamoto H., et al. "Pathogenic role of the gut microbiota in gastrointestinal disease". Intestinal Research 14 (2016): 127-138.

19. Nakae H., et al. "Gastric microbiota in the functional dyspepsia patients treated with probiotic yogurt". BMJ Open Gastroenterology (2016).

20. Öhman L., et al. "Crosstalk at the mucosal border: importance of the gut microenvironment in IBS". Nature Reviews Gastroenterology and Hepatology 12 (2015): 36-49.

21. Olivares M., et al. "Human milk composition differs in healthy mothers and mothers with celiac disease". European Journal of Nutrition 54 (2015): 119-128.

22. Rautava S., et al. "Microbial contact during pregnancy, intestinal colonization and human disease". Nature Reviews Gastroenterology and Hepatology 9 (2012): 565-576.

23. Rhee KJ., et al. "Induction of persistent colitis by a human commensal, enterotoxigenic Bacteroides fragilis, in wild-type C57BL/ 6 mice". Infection and Immunity 77 (2009): 1708-1718.

24. Rowan F., et al. "Desulfovibrio bacterial species are increased in ulcerative colitis". Diseases of the Colon and Rectum 53 (2010): 1530-1536. 
25. Rubinstein MR., et al. "Fusobacterium nucleatum promotes colorectal carcinogenesis by modulating E-cadherin/bcatenin signaling via its FadA adhesin". Cell Host and Microbe 14 (2013): 195-206.

26. Sadlack B., et al. "Ulcerative colitis-like disease in mice with a disrupted interleukin-2 gene". Cell 75 (1993): 253-261.

27. Sánchez E., et al. "Duodenal-mucosal bacteria associated with celiac disease in children". Applied and Environmental Microbiology 79 (2013): 5472-5479.

28. Sellon RK., et al. "Resident enteric bacteria are necessary for development of spontaneous colitis and immune system activation in interleukin-10-deficient mice". Infection and Immunity 66 (1998): 5224-5231.

29. Shaw SY., et al. "Association between the use of antibiotics in the first year of life and pediatric inflammatory bowel disease". The American Journal of Gastroenterology 105 (2010): 26872692.

30. Sokol H., et al. "Faecalibacterium prausnitzii is an anti-inflammatory commensal bacterium identified by gut microbiota analysis of Crohn disease patients". Proceedings of the National Academy of Sciences of the United States of America 105 (2008): 16731-16736.

31. Van Nood E., et al. "Duodenal infusion of donor feces for recurrent Clostridium difficile". The New England Journal of Medicine 368 (2013): 407-415.

32. Wright DP., et al. "Prevotella enzymes involved in mucin oligosaccharide degradation and evidence for a small operon of genes expressed during growth on mucin". FEMS Microbiology Letters 190 (2000): 73-79.

33. Zhang C., et al. "Structural resilience of the gut microbiota in adult mice under high-fat dietary perturbations". The ISME Journal 6 (2012): 1848-1857.

\section{Volume 4 Issue 6 June 2021}

\section{(C) All rights are reserved by Riddhima Banerji.}

